\title{
CANCER OF THE PROSTATE
}

\author{
By J. D. Fergusson, M.A., M.D., F.R.C.S. \\ Surgeon, St. Peter's, St. Paul's and St. Philip's Hospitals; Surgeon and Urologist, Central Middlesex Hospital; \\ Director of Teaching and Research, Institute of Urology (University of London).
}

Until comparatively recently, cancer of the prostate has failed to evoke as much interest as that accorded to other malignant conditions. This has been partly due to the rarity of its diagnosis in an early operable stage and partly because the condition commonly manifests itself at an age when active therapeutic interference has hitherto often seemed inadvisable. Both these considerations, however, have now undergone some modification. Although early diagnosis remains uncommon, new methods have been found to control the established disease, while increasing longevity and improved health among the elderly male population no longer permits the view that death from natural causes is likely to precede the complications of neoplastic extension. At the same time, the general trend towards living longer has exposed the true incidence of the disease and mortality statistics now show that it has become the commonest malignant condition affecting the male genito-urinary tract.

\section{Incidence}

Cancer of the prostate is rare before the age of fifty but increases in frequency with each subsequent decade, reaching a maximum clinical incidence between the ages of seventy and seventyfive. Thereafter, autopsy records indicate that the trend is continued, although fewer patients may actually be seen owing to their failure to survive for other reasons. A considerable difference, in fact, exists between the clinical incidence of the disease and the frequency with which histological evidence of cancer can be detected in the gland on careful examination post-mortem. The former cases are designated as showing manifest cancer while the latter, in which the disease has remained asymptomatic during life, are referred to as having latent cancer. The term occult cancer should be reserved for those in which malignant disease is suspected from the presence of atypical metastases or for other reasons; but in which the primary tumour remains unidentifiable.

The incidence of latent prostatic cancer, determined from histological examination of unselected post-mortem material, has been variously estimated at between 12 and 50 per cent. in men over fifty. Clinical or ' manifest' cancer is considerably less common but yet accounts for an annual mortality rate of about 4,000 males in England and Wales. Hospital records suggest that cancer of the prostate is seen in the ratio of one case to every 12 or 15 patients treated for urinary obstruction due to benign prostatic hypertrophy. It must be remembered, however, that the two conditions may coexist and that a small number of unsuspected or ' latent ' cancers may be brought to light following enucleation for presumed simple hypertrophy.

The distinction between latent and manifest cancer is an important one, since it implies the existence of a wide range of malignant activity among growths of similar histological appearance and suggests that this may be dependent on some extrinsic factor. Studies of the manifest disease confirm this view and point to the conclusion that growth activity may largely be controlled by endocrine influences. A recent clinical survey, in fact, has indicated that with increasing age, the frequency of metastasis declines and the disease often pursues a more protracted course.

\section{Aetiology}

No specific genetic factors are known, the condition being equally common among all races for which statistics are available. Likewise, no relationship has been established between the marital state of the individual and his liability to cancer. Various diseases of the prostate, such as chronic prostatitis and calculous disease, have, in the past, been suspected as pre-cancerous, but there is no reliable evidence to support this view. The only local abnormality commonly found in association with cancer is benign prostatic hypertrophy, the normal incidence of which, in some degree, is so great as to render an aetiological relationship difficult to establish.

\section{Pathology}

Transverse sections of the mature human prostate demonstrate the development of two distinct 
glandular zones. These comprise an inner group of glands draining by short ducts directly into the prostatic urethra, and an outer, concentric layer with longer ducts mainly converging to drain into the sulci on either side of the verumontanum. Whereas benign hypertrophy commonly arises within the inner zone, prostatic cancer practically always originates in the outer. The growth is composed of groups of cells varying in shape from cuboidal to columnar, interspersed by a greater or lesser amount of stroma. In many cases the cells appear to be arranged in the form of acini, but so great is the structural variation within an individual tumour that it is seldom possible to define it as adenocarcinomatous, scirrhous or anaplastic with accuracy. Since the initial foci of cancer are situated peripherally they are unlikely to give rise to urinary symptoms at an early stage. Subsequent local spread, however, occurs both within the substance of the gland and upwards and outwards into the region of the seminal vesicles. Direct posterior extension into the rectum is resisted by the fascia of Denonvilliers, but infiltration around the rectum with the production of a stricture is not uncommon. Downward extension to involve the external urinary sphincter is usually a late event.

Metastatic extension of the disease takes place both by the blood stream and lymphatics. Even in the early stages infiltration has been observed along the perineural lymphatics outside the gland, and post-mortem evidence suggests that involvement of the pelvic and abdominal lymph nodes is at least as common as metastasis presumed due to haematogenous spread. Characteristically, however, the metastatic disease affects the skeletal system and, on the basis of injection experiments, it seems likely that such deposits are mainly bloodborne. The bones of the pelvis and lumbar spine are commonly first involved, with further peripheral spread as the disease progresses. Isolated distant metastases occasionally occur in the early stages when the primary tumour is small and, in rare instances, may afford the first demonstrable evidence of the disease. In addition to the bones, other structures and viscera may become involved, including the lungs, liver, brain, skin, etc., and, in rare instances, the breasts and testes.

\section{Symptomatology}

In the early stages the disease remains asymptomatic. As the malignant process spreads throughout the gland it gives rise to local urinary symptoms which are generally indistinguishable from those due to benign prostatic enlargement. In addition, however, to urinary difficulty, frequency, etc., extension to the vesicles may promote haemospermia, which is regarded by some as a sign of diagnostic importance. Metastatic involvement of the bones commonly gives rise to local pain which is frequently of lumbar or sciatic distribution. Isolated metastases in other sites may give rise to local symptoms and signs and, occasionally, the superficial lymph nodes become palpably involved.

A majority of patients (about 75 per cent.) present with some form of urinary disturbance, the remainder complaining of metastatic symptoms (about 20 per cent.) or comprising those in which the disease is detected by chance during routine examination.

\section{Diagnosis}

For reasons previously mentioned, the disease is seldom detected while the growth remains confined to the periphery of the gland. Subsequent diagnosis depends primarily on the results of clinical examination, supplemented by a variety of confirmatory tests as outlined below:

\section{(a) Clinical Examination}

Rectal palpation of the prostate is of first importance in establishing the diagnosis. The characteristic features of cancer are the presence of an indurated area or nodule which contrasts with the normal consistency of the gland and which frequently distorts its contour. The affected area is often asymmetrical and may extend beyond the normal limit of the gland, particularly upwards into the vesicular region. In advanced cases the whole gland may be involved and widespread extension may occur throughout the pelvis. Where urinary obstruction is severe, retention may supervene and the distended bladder will become palpable.

Apart from the local evidence at the primary site, metastatic involvement may sometimes also give rise to clinical signs. Local tenderness may be elicited over the site of osseous deposits and secondary spread to the superficial glands in the groins and axillae may cause palpable enlargement. Involvement of the spinal cord or isolated nerves may give rise to paraplegia or local paresis. Generalized deposits in the bone marrow are often accompanied by the obvious development of anaemia. In many cases the findings on rectal examination will be unequivocal in establishing the presence of the disease. In others, however, the diagnosis, although suspected, may rem ain in doubt and necessitate the use of other methods of investigation. Even when the disease is assured, such measures may give additional information with regard to its extent.

\section{(b) Radiological Examination}

$\mathrm{X}$-ray examination of the skeleton may prove valuable, both in confirming the diagnosis and determining the degree of metastatic involvement. 


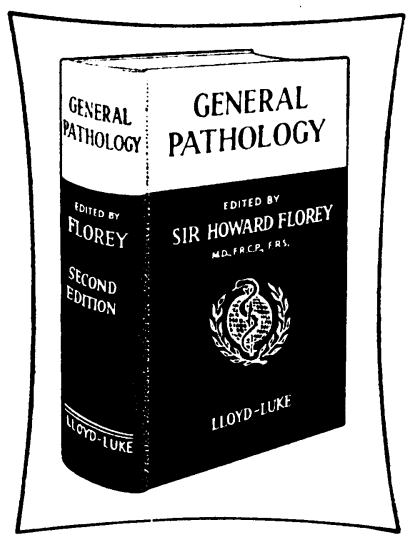

\section{Books that enshrine profound thought} GENERAL PATHOLOGY

(2nd edition) edited by SIR HOWARD FLOREY, M.D., F.R.C.P., F.R.S. Professor of Pathology, University of Oxford

The call for a second edition of this book, which appeared first under the title Lectures on General Pathology, has enabled the authors to revise its contents and add new chapters on thrombosis, metabolic changes following injury, atherosclerosis and tumours, thus enhancing its value by covering a wider field.

$x v i+932$ pp. 410 illustrations 3 colour plates (2nd edition 1958) Just published

84s. net

\section{RECENT TRENDS IN CHRONIC BRONCHITIS}

edited by NEVILLE C. OSWALD, M.D.(Cantab.), F.R.C.P.(Lond.)

Physician, St. Bartholomew's and Brompton Hospitals, London; Honorary Physician to H.M. The Queen

The main purpose of this book is to bring together under one cover for the first time recent views upon the various facets of chronic bronchitis. Much of the text consists of the original work of the several contributors. Culled from their experiences with chronic bronchitis at the Brompton Hospital since 1950, the authors present their views on the diagnosis, prevention and treatment of this distressing and often killing disease.

viii +200 pp. $\quad 76$ illustrations, 2 colour plates

(1958) 30s. net

Just published

\section{LLOYD-LUKE (Medical Books) LTD., 49 Newman Street, W.I}

Characteristically, the bone lesions are osteosclerotic in nature and thus differ from other secondary deposits of a similar nature. Metastasis most commonly takes place earliest in the bones adjacent to the prostate, and thus the pelvis, lumbar spine and upper femora should be examined. About 20 per cent. of cases suspected on cilnical grounds of having prostatic cancer show radiological evidence of bone involvement at the outset. On the other hand, negative radiological findings do not necessarily exclude metastasis, a proportion of cases with clinical evidence of involvement remaining unconfirmed at the initial $\mathrm{X}$-ray examination.

\section{(c) Serum Acid Phosphatase Estimation}

This test has little primary diagnostic importance, since in practically all cases where the value is significantly raised, the presence of the disease will already have been established beyond much doubt. As a confirmatory measure it may be reassuring and, when repeated, it is sometimes useful as an index of the response to endocrine therapy. Determination of the serum acid phosphatase by the King Armstrong technique, using formalin to inactivate any enzyme derived from extra-prostatic sources, shows a normal range of up to 2.5 units. Values exceeding these are suggestive of prostatic cancer and figures of over 5 units are almost certainly confirmatory. About 75 per cent. of cases with demonstrable bone metastases show a raised serum value, the proportion falling to about 30 per cent. in apparently nonmetastasizing cases.

\section{(d) Biopsy}

Final confirmation of the presence of cancer can only be assured by histological examination. Small portions of tissue may be obtained from the primary site, either by perurethral resection, needle (or open) biopsy via the perineum or transrectal biopsy. The indications for prostatic biopsy are: (a) as a prelude to radical surgery (in the rare early cases where a suspicious nodule is found on rectal examination); (b) to confirm the diagnosis in other suspected though unproven cases; and (c) as an integral part of prostatic resection for the relief of urinary obstruction. It should be emphasized with regard to (a) that the presence of histological cancer provides no index of its activity, whereas in the symptomatic disease a positive finding may be regarded as of definite significance. Biopsy may also be undertaken on secondary deposits where these are reasonably 
accessible, as in the case of superficial glandular involvement, skin nodules, and in certain bones.

\section{(e) Cytological Examination of the Prostatic Secretion}

In cases of prostatic cancer, cytological examination of the prostatic fluid, expressed per urethram after rectal massage of the gland, will often reveal clumps of malignant cells. Positive results are, however, relatively infrequent in cases where the disease is not already suspected on rectal palpation and the test is, therefore, mainly of confirmatory value. It is unknown whether a positive finding indicates only the presence of actively invasive cancer or whether a similar result can be obtained in cases with a small, latent peripheral focus.

\section{Treatment}

(a) Radical surgery is the only form of treatment offering any prospect of complete cure of the disease. In order to assess its applicability, however, it is necessary to draw attention to the following points:-

(i) In present circumstances, early diagnosis is unusual and the condition is rarely detected in an operable phase.

(ii) The chance findings of a suspicious nodule on rectal palpation in an asymptomatic patient does not necessarily imply the presence of active disease, even though cancer is reported at biopsy.

(iii) The clinical detection of an apparently isolated cancerous nodule does not exclude the possibility of extra-prostatic lymphatic extension.

(iv) Radical prostatectomy represents a major surgical procedure and cannot be divorced from a certain operative mortality and post-operative morbidity. In the age group mainly affected by the disease a certain proportion will be unfit for operation.

(v) In the opinion of many, the results of endocrine control therapy combined, if necessary, with palliative surgery compare favourably with those of the radical operation.

Consideration of these factors indicates that, although radical extirpation may afford the only certain method of eliminating the disease, there are comparatively few cases in which it is applicable and none in which the outcome can confidently be assured. Evidence shows, however, that when cancer of the prostate occurs below the age of sixty it frequently runs a more active course and, in these circumstances, if detected in an operable phase, radical surgery should certainly be contemplated. Somewhat less than 5 per cent. cases will fall into this category and the remainder will require treatment on the lines given below.

(b) Endocrine control therapy. It is now well established that most cases of prostatic cancer are susceptible to hormonal influence. Deprivation of androgens (by castration) or the administration of oestrogens brings about a comparable diminution of malignant activity which is often reflected in a shrinkage of the primary growth and the relief of metastatic symptoms. At the same time, objective evidence of improvement may be afforded by a lowering of the serum acid phosphatase, changes in the radiological appearance of bone deposits, and alteration in the histological structure of the tumour.

(i) Castration is best performed by the simple method of bilateral subcapsular orchidectomy. A single horizontal scrotal incision will expose both testes, whereupon the tunica albuginea of each is incised and the functional tissue removed by blunt dissection. The rationale of treatment is to remove the main depots of androgen elaboration and thus to reduce the stimulating effect on the tumour.

(ii) Oestrogen therapy depends on the administration of an oestrogenic hormone with the prime object of reducing pituitary gonadotrophic activity and thus removing the stimulus to androgen production. At the same time it has been claimed that the oestrogen may exert a direct cytotoxic effect on the growth itself. The oral administration of stilboestrol or one of its synthetic counterparts, in doses of $\mathrm{I}_{5}$ to $100 \mathrm{mg}$. o.d., is often effective in combatting the activity of the malignant process for a time, but the method has the disadvantage of inducing certain side effects irrespective of the dosage employed. These, in the main, consist of feminizing changes such as enlargement of the breasts, etc., but nausea and vomiting may occasionally be troublesome. The use of substituted natural oestrogens is said :to minimize such disturbances, but their oral administration is less effective in promoting growth control. Recently the use of phosphorylated oestrogen has been advocated either by intravenous injection or oral administration. This substance is stated to be inert until it is broken down by phosphatase with the liberation of free oestrogen. The claim is made that it becomes active only at the sites of phosphatase elaboration and thus exerts a concentrated cytotoxic effect on the tumour. In this way the production of side effects elsewhere is said to be reduced. Insufficient scientific evidence is at present available to support this contention, but it seems fair to state that the preparation sometimes appears to be effective when other oestrogens have failed. This may in part be due to the high dosage advocated.

Despite the fact that both oestrogen therapy and castration exert a favourable effect on the symptomatology of the disease, and on the period of survival, many cases ultimately relapse and become resistant to further treatment. At this stage it may 
be considered that, while some tumours become altogether hormone-independent, others are reactivated by a compensatory production of androgen from extra-genital sources. Chief among the latter appear to be the adrenal glands which are known often to undergo hyperplasia after castration.

(iii) Adrenalectomy may be carried out in cases relapsing after castration or oestrogen therapy, the patients well-being being maintained by cortisone substitution. A further measure of tumour control may thereby often be achieved, but, in general, in elderly patients the expected period of natural survival is too short to warrant their submission to the operation with its subsequent regime. Nevertheless, striking symptomatic improvement may occur in favourable cases and survival in comfort for two to three years, with objective evidence of growth regression, has been reported. Cortisone therapy alone, with the object of suppressing adrenal activity, has so far seemed of little avail.

(iv) Hypophysectomy. Inactivation of the pituitary, either by surgical ablation or irradiation, has the object of eliminating gonadotrophic activity and thus depressing androgen production. Surgical hypophysectomy is seldom applicable to the advanced relapsing stage of the disease and would seem to be too radical in earlier cases which might well respond to oestrogen therapy. Irradiation hypophysectomy, however, is a simpler procedure, imposing no great strain or inconvenience on the relapsing case, and is carried out by the introduction of radioactive yttrium into the pituitary by the transnasal route under $\mathrm{X}$-ray control. The method is still somewhat in an experimental phase and the induction of complete radionecrosis of the gland is uncertain. Evidence of renewed tumour control has followed its use but continued cortisone substitution is required.

While the above methods of endocrine control are effective in most instances in securing regression of the disease, adjuvant measures are sometimes required for relieving urinary retention and combatting residual symptoms.

(c) Palliative surgery may be needed in cases of urinary obstruction when a combination of endocrine therapy and simple catheterization fail to bring about relief within a reasonable time. Perurethral resection is the method of choice and is much to be preferred to suprapubic drainage after which a permanent fistula is likely to result.

(d) Radiotherapy has been for the most part unsatisfactory in the treatment of the disease. Most growths are relatively insensitive to irradiation and the delivery of an effective dose of X-rays leads to destruction or irritation of the surrounding normal tissues. The direct implantation of radium, radon seeds and, more recently, radioactive gold or yttrium into the primary tumour has led to similar consequences. Improved results may perhaps be expected with supervoltage apparatus and there is no reason why such therapy should not be combined with other forms of treatment. The limitations of radiotherapy as applied to the metastasizing disease will be obvious, although palliation of local metastatic pain is occasionally worthwhile.

(e) Palliative medical treatment consists in the employment of routine urinary antiseptics to control urinary infection and the use of suitable analgesics or hypnotics for the relief of metastatic pain. Blood transfusion is seldom indicated for anaemia, since the latter is usually a manifestation of diffuse involvement of the bone marrow in the terminal stages of the disease.

\section{Prognosis}

The outlook in prostatic cancer can be reviewed in terms of the symptomatic course and period of survival. There is no doubt whatever that the former has been vastly improved by the introduction of endocrine therapy and it is nowadays rare to witness the protracted discomfort which used to characterize the progress of the disease. The duration of survival, however, does not appear to have been influenced to a corresponding extent. Taking all cases into account, including those who first present with widespread cancer, the mortality rate at the end of a year amounts to nearly 50 per cent. Thereafter the rate diminishes with an average overall survival from diagnosis to death of between 30 and 36 months. The presence of demonstrable metastases when the patient is first seen naturally affects the prognosis adversely. No particular relationship can be discerned between the serum acid phosphatase value and the sensitivity of the tumour to endocrine therapy, but the former may sometimes be of value in assessing the response to treatment. In general, the disease appears to run a more active course in younger patients while, conversely, it is not unusual for an elderly case to survive for several years with minimal supervision. 